\title{
Modelagem de efeitos genéticos e ambientais que influenciam o peso à desmama de bezerros mestiços Charolês-Zebu ${ }^{1}$
}

\author{
Fábio Luiz Buranelo Toral², Roberto Augusto de Almeida Torres Júnior ${ }^{3}$, Paulo Sávio Lopes ${ }^{4}$, \\ Luiz Otávio Campos da Silva ${ }^{3}$
}

\author{
1 Apoio financeiro da FAPEMIG (PPM-00456-11) \\ 2 Universidade Federal de Minas Gerais. \\ ${ }^{3}$ Embrapa Gado de Corte. \\ ${ }^{4}$ Universidade Federal de Viçosa. Bolsista de Produtividade em Pesquisa do CNPq.
}

RESUMO - Este trabalho foi realizado com o objetivo de avaliar alternativas para modelagem de efeitos genéticos e ambientais que influenciam o peso à desmama de bezerros mestiços Charolês-Zebu. Foram utilizados os dados de peso à desmama de 56.965 bezerros, com percentuais de alelos de origem da raça Charolesa variando entre $23 \%$ e $66 \%$. Os modelos considerados diferiram quanto à inclusão de efeitos genéticos aditivos e não-aditivos médios, individuais e maternos, bem como quanto à maneira de modelar estes efeitos (regressão múltipla ou variável classificatória). Também foi avaliada a inclusão da interação entre o grupo genético do bezerro e seu grupo de contemporâneos, como um efeito aleatório não correlacionado. De acordo com os critérios de ajuste utilizados, a interação entre o grupo genético e o grupo de contemporâneos representa parte significativa da variação observada para o peso à desmama de bezerros Charolês-Zebu e os efeitos dos percentuais de alelos de origem da raça Charolesa e de heterozigoses, individuais e maternos, podem ser modelados por meio da utilização de regressão múltipla.

Palavras-chave: avaliação genética multirracial, bovinos de corte, cruzamento, heterozigose, modelo estatístico

\section{Modeling of genetic and environmental effects affecting the weaning weight of Charolais-Zebu crossbred calves}

\begin{abstract}
This study was carried out to evaluate some alternatives for modeling of genetic and environmental effects influencing the weaning weight of Charolais-Zebu crossbred calves. The weaning weight data of 56,965 calves with alleles' percentage from the Charolais breed varying from $23 \%$ to $66 \%$ were used. The models under comparison differed for the inclusion of the individual and maternal additive and non-additive genetic effects, as well as the way to model those effects (multiple regression or discrete variable). The inclusion of the interaction between the calf genetic group and its contemporary group was also evaluated as a random uncorrelated effect. The adjustment criteria pointed out the interaction between the calf genetic group and its contemporary group to represent a significant part of the variation observed for the weaning weight of the Charolais-Zebu crossbred calves, and the effects of alleles' percentage from the Charolais breed and the heterozygosity, individual and maternal, can be modeled by using the multiple regression.
\end{abstract}

Key Words: beef cattle, crossbreeding, heterozygosis, multiple-breed genetic evaluation, statistical model

\section{Introdução}

O melhoramento genético pode contribuir para melhoria dos índices produtivos da bovinocultura de corte por meio da seleção de reprodutores geneticamente superiores e dos cruzamentos. Nos cruzamentos, além da possibilidade de aproveitamento dos benefícios provenientes da seleção dos indivíduos dentro das raças, é possível explorar a heterose e a complementaridade. Quando o cruzamento tem por objetivo a formação de uma raça composta, a seleção também deve ser realizada nas populações de animais mestiços e a avaliação genética é uma ferramenta importante para identificação do potencial genético dos animais para as características de interesse.

Nas avaliações genéticas intrarraciais de bovinos, para características de crescimento, efeitos genéticos aditivos direto e materno, de ambiente materno permanente, de grupos de contemporâneos e das idades do bezerro e da mãe são, geralmente, considerados (Mercadante \& Lôbo, 1997; Paz et al., 1999; Silveira et al., 2004). Contudo, em avaliações de populações cruzadas, é necessário que os efeitos aditivos e não-aditivos médios, que podem ser fontes significativas de variação (Newman et al., 1993; Trematore et al., 1998; Roso \& Fries, 2000) também sejam modelados 
adequadamente para que os animais de diferentes grupos genéticos possam ser comparados. Portanto, este trabalho foi realizado com o objetivo de avaliar alternativas para modelagem de efeitos genéticos e ambientais que influenciam a expressão do peso à desmama de bezerros mestiços Charolês-Zebu.

\section{Material e Métodos}

Os dados utilizados neste trabalho foram fornecidos pela Associação Brasileira de Criadores de Canchim (ABCCAN) e incluíram os dados genealógicos e os pesos à desmama de bezerros mestiços Charolês-Zebu, envolvidos na obtenção de animais da raça Canchim (ABCCAN, 2008).

$\mathrm{O}$ arquivo de dados analisado foi composto por 56.965 observações de pesos à desmama (225 dias de idade), de bezerros nascidos entre janeiro de 1988 e fevereiro de 2005, filhos de 1.600 touros e de 27.122 vacas que pariram entre 2 e 15 anos de idade, netos de 1.929 avôs maternos, distribuídos em 4.458 grupos de contemporâneos de 247 fazendas, localizadas nas regiões Sul (RS, SC e PR), Sudeste (SP, RJ e MG), Centro-Oeste (MS, MT, GO e DF), Nordeste (BA, PE, PI e MA) e Norte (TO e PA). A matriz de parentesco foi composta por 87.312 animais.

Os grupos de contemporâneos foram formados pela concatenação das variáveis: fazenda, ano e época de nascimento (época 1 - entre setembro e novembro; época 2 - entre dezembro e fevereiro do ano seguinte; época 3 entre março e maio; e época 4 - entre junho e agosto), sexo e regime alimentar do bezerro à desmama (RA 1 - pasto; RA 2 - pasto adubado; RA 3 - pasto adubado e pastejo rotacionado; RA 4 - pasto irrigado; RA 5 - suplementado à pasto; e RA 6 - confinado).

Outras informações sobre a edição do arquivo de dados e de parentesco, cálculos dos percentuais esperados de alelos de origem Charolesa e de heterozigose e sobre os cruzamentos analisados podem ser obtidas em Toral et al. (2009, 2010).

Para modelagem dos efeitos das composições genéticas individuais e maternas por modelos classificatórios, optouse pela utilização de uma definição alternativa para os grupos genéticos, o que permitiu a diferenciação de animais considerados do mesmo grupo, segundo as definições da ABCCAN, mas que são provenientes de diferentes esquemas de cruzamento, especialmente em função de diferenças raciais e heteróticas existentes entre suas mães. Esta definição levou à criação de 12 classes de grupos genéticos (Tabela 1).

Foram avaliados sete modelos estatísticos para o peso à desmama, utilizando-se a metodologia de Modelos Mistos (Henderson et al., 1959). O primeiro modelo considerado foi o mais simples (MSIM) e pode ser representado pela equação $P 225_{i j l m}=\mu+a_{i}+m_{j}+p_{j}+G C_{l}+c_{1_{(s)}} I V P_{m}+c_{2(s)} I V P_{m}^{2}+c_{3_{(s)}}$ $\left(\max \left(0, I V P_{m}-6,33\right)\right)^{2}+c_{4_{(\xi)}}\left(\max \left(0, I V P_{m}-10,66\right)\right)^{2}+d_{1_{(\xi)}}\left(P C_{j} \times I V P_{m}\right)$ $+d_{2_{(s)}}\left(P C_{j} \times I V P_{m}\right)^{2}+d_{3_{(s)}}\left(P C_{j} \times\left(\max \left(0, I V P_{m}-6,33\right)\right)\right)^{2}+$ $d_{4_{(\xi)}}\left(P C_{j} \times\left(\max \left(0, I V P_{m}-10,66\right)\right)\right)^{2}+e_{i j / m}$

em que: $P 225_{i j l m}=$ peso à desmama, padronizado para 225 dias de idade, do animal $i$, filho da vaca $j$ com idade $m$, criado no grupo de contemporâneos $l ; \mu=$ constante geral; $a_{i}=$ efeito genético aditivo direto do animal $i ; m_{j}=$ efeito genético aditivo materno da vaca $j ; p_{j}=$ efeito de ambiente materno permanente da vaca $j ; G C_{l}=$ efeito do grupo de contemporâneos $l(l=1, \ldots, 4.458) ; c_{h_{(s)}}=h^{e ́ s i m o}$ coeficiente de regressão $(h=1,2,3,4)$ para a idade da vaca ao parto, independentemente do percentual de Charolês da vaca, aninhado em sexo $s$ do bezerro ( $s=$ macho e fêmea); $I V P_{m}$, a idade $m(m=2,00, \ldots, 15,00$ anos) da vaca ao parto; $d_{g_{(s)}}=g^{\text {ésimo }}$ coeficiente de regressão $(g=1,2,3,4)$ para a idade da vaca ao parto dependente do percentual de Charolês

Tabela 1 - Médias dos percentuais de alelos da raça Charolesa (PC) e de heterozigose (PH) dos bezerros e vacas e número de observações (N) para cada grupo genético

\begin{tabular}{|c|c|c|c|c|c|c|}
\hline \multicolumn{2}{|c|}{ Grupo genético ${ }^{1}$} & \multicolumn{2}{|c|}{ Bezerro } & \multicolumn{2}{|c|}{ Vaca } & \multirow[b]{2}{*}{$\mathrm{N}$} \\
\hline Bezerro & Vaca & $\mathrm{PC}$ & $\mathrm{PH}$ & $\mathrm{PC}$ & $\mathrm{PH}$ & \\
\hline $1 / 4 \mathrm{Ch}$ & T1 (15/32 Ch) & 0,2344 & 0,4688 & 0,4688 & 0,5469 & 7 \\
\hline A (5/16 Ch) & Canchim & 0,3081 & 0,6162 & 0,6162 & 0,4728 & 20 \\
\hline T1 (15/32 Ch) & A (5/16 Ch) & 0,4664 & 0,5451 & 0,3126 & 0,6253 & 124 \\
\hline V (9/16 Ch) & T1 (15/32 Ch) & 0,5457 & 0,5076 & 0,4687 & 0,5468 & 252 \\
\hline Canchim & V (9/16 Ch) & 0,5886 & 0,4860 & 0,5595 & 0,5010 & 4.973 \\
\hline Canchim & $5 / 8 \mathrm{Ch}$ & 0,6239 & 0,4694 & 0,6250 & 0,7500 & 1.124 \\
\hline Canchim & Canchim & 0,6190 & 0,4716 & 0,6177 & 0,4722 & 32.631 \\
\hline Canchim & MA (21/32 Ch) & 0,6462 & 0,4575 & 0,6562 & 0,6877 & 5.680 \\
\hline MA (21/32 Ch) & A (5/16 Ch) & 0,6562 & 0,6877 & 0,3123 & 0,6247 & 10.569 \\
\hline
\end{tabular}

${ }^{1} \mathrm{Ch}=$ Charolês; $\mathrm{Z}=$ Zebu. 
da vaca, aninhado em sexo $s ; P C_{j}=$ percentual de alelos de origem da raça Charolesa da vaca $j$; e $e_{i j l m}=$ erro aleatório associado a cada observação. Os coeficientes $c_{h_{(s)}}$ foram usados para modelar uma curva de maturação e senescência para as vacas Zebuínas $\left(P C_{j}=0\right)$, com um polinômio segmentado linear + quadrático - quadrático - quadrático com nós aos 6,33 e 10,66 anos de idade, enquanto os coeficientes $d_{g_{(s)}}$ foram usados na modelagem das diferenças entre as curvas de maturação e senescência de vacas Charolesas, em relação às das vacas Zebuínas. Assim, para as vacas mestiças, as curvas podem ser obtidas pela combinação dessas duas curvas, proporcionalmente à porcentagem de alelos de origem da raça Charolesa do grupo de interesse.

No segundo modelo (MSIM + INT), foram incluídos os efeitos do modelo MSIM e o efeito aleatório não correlacionado da interação entre o grupo genético e o grupo de contemporâneos dos bezerros (GG $\times G C_{q}, q=1$, ..., 9.013), numa tentativa de modelar, pelo menos parcialmente, um efeito de interação genótipo $\times$ ambiente e, ou, diferenças no manejo dado a animais contemporâneos de grupos genéticos diferentes.

No terceiro modelo (MSIM + RM), além dos efeitos considerados no modelo MSIM, foram incluídos os coeficientes de regressão lineares para modelar os efeitos dos percentuais de alelos da raça Charolesa do animal $i$, $b_{1}\left(P C_{i}\right)$, e da vaca $j, b_{2}\left(P C_{j}\right)$, e os percentuais de heterozigose do animal $i, b_{3}\left(\mathrm{PH}_{i}\right)$, e da vaca $j, b_{4}\left(\mathrm{PH}_{j}\right)$.

No quarto modelo (MSIM + CGG), considerou-se o efeito classificatório fixo do grupo genético do animal $\left(G G_{t}, t=1, \ldots, 12\right)$ e os demais efeitos incluídos no modelo MSIM. Este modelo possibilita considerar os efeitos raciais e heteróticos em conjunto, mas de forma aproximada, porque nem todos os animais de um grupo genético têm exatamente a mesma composição em termos de percentuais de alelos da raça Charolesa e de heterozigoses individuais e maternos.

No quinto modelo (MSIM + RM + INT), foram incluídos aqueles efeitos do modelo (MSIM + RM) e a interação $G G \times G C_{q}$. No sexto modelo (MSIM + CGG + INT), além dos efeitos considerados no modelo MSIM + CGG, foi incluída a interação $G G \times G C_{q}$. Também foi considerado um modelo utilizado para avaliação genética da raça Canchim (MUTIL), que é semelhante ao modelo MSIM, mas com o efeito do grupo genético do animal participando da formação do grupo de contemporâneos (ABCCANEmbrapa-Geneplus, 2004).

As pressuposições assumidas para os efeitos aleatórios foram

$$
\left[\begin{array}{c}
a \\
m \\
p \\
q \\
e
\end{array}\right] \sim N M V\left\{\left[\left[\begin{array}{l}
0 \\
0 \\
0 \\
0 \\
0
\end{array}\right] ;\left[\begin{array}{ccccc}
G & B & 0 & 0 & 0 \\
B & M & 0 & 0 & 0 \\
0 & 0 & P & 0 & 0 \\
0 & 0 & 0 & Q & 0 \\
0 & 0 & 0 & 0 & R
\end{array}\right]\right\},\right.
$$

em que: $G$ = matriz de covariâncias entre os efeitos genéticos aditivos diretos, $G=A \sigma_{a}^{2} ; A=$ matriz de parentesco; $\sigma_{a}^{2}=$ variância genética aditiva direta; $M=$ matriz de covariâncias entre os efeitos genéticos aditivos maternos, $M=A \sigma_{m}^{2} ; \sigma_{m}^{2}=$ a variância genética aditiva materna; $B=$ matriz de covariâncias entre os efeitos genéticos aditivos diretos e maternos, $B=A \sigma_{a m} ; \sigma_{a m}=$ covariância entre os efeitos genéticos aditivos direto e materno; $P=$ matriz de covariâncias dos efeitos de ambiente materno permanente, $P=I_{N v} \sigma_{p}^{2} ; I_{N v}=$ matriz identidade de ordem Nv; $\sigma_{p}^{2}=$ variância de ambiente materno permanente; $Q=$ matriz de covariâncias dos efeitos da interação $G G \times G C, Q=I_{N i} \sigma_{q}^{2}$; $I_{N i}=$ matriz identidade de ordem $\mathrm{Ni} ; \sigma_{q}^{2}=$ variância atribuída a interação entre grupo genético e grupo de contemporâneos; $R$ = matriz de covariâncias entre os resíduos, $R=I_{N} \sigma_{e}^{2} ; \quad I_{N}=$ matriz identidade de ordem $\mathrm{N}$; e $\sigma_{e}^{2}=$ variância residual.

Os componentes de variância foram estimados pelo método da Máxima Verossimilhança Restrita. As estimativas foram utilizadas na resolução do sistema de equações de Modelos Mistos para obtenção das soluções para os efeitos fixos e aleatórios, utilizando-se o programa MTDFREML (Boldman et al., 1995). O critério de convergência foi alcançado quando a variância dos valores assumidos pela verossimilhança nos pontos do Simplex foi inferior a $10^{-9}$. As análises foram reiniciadas até que o valor da função de verossimilhança restrita estivesse constante.

A comparação entre os modelos foi realizada em dois estágios, conforme recomendado por Wolfinger (1993): primeiro estágio, foram avaliadas as melhorias nos ajustes proporcionadas pela inclusão da interação $G G \times G C$ (MSIM + INT $\times$ MSIM, MSIM + RM + INT $\times$ MSIM + RM e MSIM + CGG + INT $\times$ MSIM + CGG). Neste caso, a estatística do Teste da Razão de Verossimilhança (LRT) pode ser calculada como a diferença entre os valores assumidos pelo dobro do logaritmo da função de verossimilhança restrita $(-2 \mathrm{Ln}(\mathrm{L}))$ de dois modelos. O valor do $-2 \mathrm{Ln}(\mathrm{L})$ foi obtido como

$$
\begin{aligned}
& -2 \operatorname{Ln}(L)_{x}=\left(N-p_{x}\right) \times \operatorname{Ln}(2 \pi)+\operatorname{Ln}|V|+\operatorname{Ln}\left|X^{\prime} V^{-1} X\right|+ \\
& +(y-X \beta)^{\prime} V^{-1}(y-X \beta),
\end{aligned}
$$

em que: $-2 \operatorname{Ln}(L)_{X}=$ valor da função de verossimilhança restrita do modelo $x ; \mathrm{N}$ = número de observações utilizadas; $p_{x}=$ posto da matriz de delineamento do modelo $x$ e serve para considerar a redução dos graus de liberdade, em 
função da estimação das soluções para os efeitos fixos do modelo; e $V=$ matriz de covariância fenotípica (Wolfinger, 1993; Boldman et al., 1995). Para determinação do nível de significância observado, a estatística do LRT foi comparada ao valor obtido da distribuição acumulada de qui-quadrado, com graus de liberdade igual à diferença no número de efeitos aleatórios considerados.

O Critério de Informação de Akaike (AIC) e o AIC Consistente (CAIC) também foram calculados. Wolfinger (1993) ressalta que estes últimos critérios são mais conservadores que o LRT porque consideram o número de efeitos aleatórios considerados nos modelos. As fórmulas utilizadas para cálculo desses critérios foram

$$
\begin{aligned}
& A I C_{x}=-2 \operatorname{Ln}(L)_{x}+2 k_{x}, \mathrm{e} \\
& C A I C_{x}=-2 \operatorname{Ln}(L)_{x}+k_{x}\left[\operatorname{Ln}\left(N-p_{x}\right)+1\right],
\end{aligned}
$$

em que: $A I C_{x}=$ critério de informação de Akaike do modelo $x$; $k_{x}=$ número de componentes de covariância do modelo $x$; e $C A I C_{X}=$ critério de informação de Akaike consistente do modelo $x$ (Bozdogan, 1987; Wolfinger, 1993). No caso dos critérios AIC e CAIC, foram comparados os valores absolutos, conforme apresentado por Bozdogan (1987).

No segundo estágio, os modelos foram comparados quanto aos ajustes proporcionados pela inclusão dos diferentes efeitos fixos. Neste caso, os valores de -2Ln(L) podem não ser adequados para comparação de modelos, pois, com a utilização do REML, os efeitos fixos são 'eliminados' (Wolfinger, 1993). Os critérios AIC e CAIC foram recalculados conforme a sugestão de Wolfinger (1993), por meio das equações:

$$
\begin{aligned}
& A I C_{x}=-2 \operatorname{Ln}(L)_{x}+2\left(p_{x}+k_{x}\right), \mathrm{e} \\
& C A I C_{x}=-2 \operatorname{Ln}(L)_{x}+\left(p_{x}+k_{x}\right)[\operatorname{Ln}(N)+1] .
\end{aligned}
$$

\section{Resultados e Discussão}

Os níveis de significância obtidos no teste de razão de verossimilhança (Tabela 2) apontam para a rejeição da hipótese $H_{0}: \sigma_{q}^{2}=0$; portanto, a inclusão da interação entre grupo genético e grupo de contemporâneos proporcionou melhoria significativa no ajuste dos modelos, independentemente dos demais efeitos fixos considerados. Este resultado corrobora a importância da interação genótipo $\times$ ambiente, conforme demonstrado por Toral et al. (2004), Alencar et al. (2005) e Carvalheiro et al. (2006). A utilização de um modelo que não contempla a interação genótipo $\times$ ambiente poderia provocar alterações nas soluções para os efeitos fixos (Carvalheiro et al., 2006) e nos componentes de covariância (Alencar et al., 2005), o que poderia reduzir a eficiência de identificação dos animais geneticamente superiores e reduzir o progresso genético.

Neste caso, essa interação significativa entre grupos genéticos e grupos de contemporâneos poderia ser explicada por uma interação genótipo $\times$ ambiente típica, em que as diferenças entre os genótipos dependem dos ambientes considerados. A adoção de alternativas de manejo específicas para cada grupo genético, especialmente manejo nutricional, também pode ser uma das causas desta interação.

Com relação à modelagem dos efeitos fixos, os critérios de ajuste (-2Ln(L) e AIC) proporcionados pelo modelo utilizado na avaliação genética para peso à desmama dos animais da raça Canchim (MUTIL) foram os mais próximos de zero, entre aqueles avaliados, e sugerem melhor ajuste do modelo citado.

No modelo MUTIL, o efeito de grupo genético é considerado parte do grupo de contemporâneos, portanto,

\begin{tabular}{|c|c|c|c|c|c|c|c|}
\hline Modelo & $-2 \mathrm{Ln}(\mathrm{L})$ & AIC & CAIC & $\mathrm{k}$ & $\mathrm{p}(\mathrm{X})$ & MR & $\mathrm{p}$ \\
\hline & \multicolumn{7}{|c|}{ Primeiro estágio - efeito aleatório da interação grupo genético $\times$ grupo de contemporâneos } \\
\hline MSIM & $405.130,4$ & $405.140,4$ & $405.189,7$ & 5 & 4.474 & - & - \\
\hline MSIM + INT & $404.909,7$ & $404.921,7$ & $404.980,9$ & 6 & 4.474 & MSIM & $<0,01$ \\
\hline MSIM + RM & $404.851,7$ & $404.861,7$ & $404.863,6$ & 5 & 4.478 & - & - \\
\hline MSIM + CGG & $404.722,9$ & $404.732,9$ & $404.782,2$ & 5 & 4.485 & - & - \\
\hline MSIM + RM + INT & $404.660,5$ & $404.672,5$ & $404.731,7$ & 6 & 4.478 & MSIM + RM & $<0,01$ \\
\hline MSIM + CGG + INT & $404.552,3$ & $404.564,3$ & $404.623,5$ & 6 & 4.485 & MSIM + CGG & $<0,01$ \\
\hline
\end{tabular}
não permitindo que os desempenhos dos diferentes grupos

Tabela 2 - Estatísticas ${ }^{1}$ para comparação de modelos quando à presença da interação grupo genético × grupo de contemporâneos e quanto às alternativas para modelagem dos efeitos genéticos aditivos e não-aditivos médios

Segundo estágio - forma de ajuste dos efeitos genéticos aditivos e não-aditivos médios ${ }^{2}$

MSIM + INT

$\mathrm{MSIM}+\mathrm{RM}+\mathrm{INT}$

$\mathrm{MSIM}+\mathrm{CGG}+\mathrm{INT}$

MUTIL

$413.869,7$
$413.628,5$
$413.534,3$
$389.835,8$

$404.909,7$

$404.660,5$

$404.552,3$

$371.767,8$

$\begin{array}{lll}458.446,6 & 6 & 4.474 \\ 458.245,2 & 6 & 4.478 \\ 458.220,6 & 6 & 4.485 \\ 479.726,8 & 5 & 9.029\end{array}$

\footnotetext{
${ }^{1}$ Valores da função de verossimilhança restrita (-2Ln(L)), do critério de informação de Akaike (AIC) e do AIC consistente (CAIC), número de componentes de covariância do modelo (k), posto da matriz de delineamento $(\mathrm{p}(\mathrm{X})$ ), modelos de referência para os testes da razão de verossimilhança (MR) e níveis de significância dos testes (p). 2 Valores de AIC e CAIC recalculados conforme as sugestões de Wolfinger (1993).
} 
genéticos sejam estimados e comparados. A utilização deste modelo implica menor aproveitamento dos dados disponíveis porque a concatenação da variável grupo genético ao grupo de contemporâneos leva à formação de grupos de contemporâneos menores e ao aumento do número de grupos de contemporâneos com apenas uma observação. Considerando o critério CAIC, o modelo de melhor ajuste foi aquele que contemplou os efeitos de grupo genético (classes) e a interação entre grupo genético e grupo de contemporâneos (MSIM + CGG + INT), seguindo-se pelos modelos MSIM + RM + INT, MSIM + INT e MUTIL. O ajuste proporcionado pelo modelo que utilizou regressão múltipla para modelagem dos efeitos genéticos aditivos e nãoaditivos médios e a interação do grupo genético com o grupo de contemporâneos (MSIM + RM + INT) foi melhor que o proporcionado pelo modelo semelhante que não considerou esses efeitos genéticos (MSIM + INT), como indicaram os valores de $-2 \mathrm{Ln}(\mathrm{L})$, AIC e CAIC. Como a diferença entre esses dois modelos é a presença dos coeficientes $b_{1}, b_{2}, b_{3}$ e $b_{4}$, pode-se inferir que a hipótese $H_{0}: b_{1}=b_{2}=b_{3}=b_{4}=0$ deve ser rejeitada. As estimativas de $b_{1}, b_{2}, b_{3}$ e $b_{4}$, obtidas por meio da metodologia de Modelos Mistos, no modelo que também contemplou a interação entre grupo genético e grupo de contemporâneos foram: 35,973; 11,695; 96,443; e 28,190 kg, respectivamente. Estes resultados estão de acordo com aqueles obtidos por outros autores (Trematore et al., 1998; Roso \& Fries, 2000; Carvalheiro et al., 2006; Pimentel et al., 2006).

Com relação ao valor positivo estimado para o coeficiente $b_{1}$, utilizado para modelar o efeito genético aditivo individual médio, concorda-se com a explicação de Trematore et al. (1998) de que valores positivos seriam esperados, em razão das características dos dois grupos de raças utilizadas nos cruzamentos, e indicam efeito positivo dos alelos da raça Charolesa para o aumento do peso à desmama. A Charolesa é uma raça de grande porte, com elevado potencial para crescimento, enquanto as raças zebuínas são menores e apresentam menores potenciais para crescimento (Trematore et al., 1998). Por outro lado, uma estimativa positiva para $b_{2}$ contraria a expectativa de Trematore et al. (1998), que encontraram estimativa negativa para este coeficiente e atribuíram isto ao fato de as vacas com maior percentual de alelos da raça Charolesa apresentarem maiores exigências nutricionais que, nas condições em que os dados utilizados por eles foram obtidos, não poderiam ser completamente atendidas. Neste caso, a média dos percentuais de alelos da raça Charolesa das vacas nos grupos de contemporâneos com regimes alimentares diferentes de pasto foram maiores que a média desses percentuais das vacas nos grupos com regime alimentar de pasto, sugerindo uma preocupação dos criadores no sentido de fornecer melhores condições alimentares para as vacas mais exigentes e, pelo menos parcialmente, contribuindo para explicar a obtenção de uma estimativa positiva para $b_{2}$.

As estimativas dos coeficientes de regressão dos percentuais de heterozigoses diretas e maternas também foram positivas, confirmando os resultados de Trematore et al. (1998) e de Roso \& Fries (2000), indicando a importância de efeitos genéticos não-aditivos (heterozigose) sobre características de crescimento (no caso da heterozigose individual) e sobre a habilidade materna (no caso da heterozigose materna). Os resultados obtidos por Cerdótes et al. (2004) ajudam a explicar o efeito positivo da heterozigose materna sobre o peso à desmama. Esses autores verificaram que a produção de leite das vacas F1 Charolês-Nelore, que apresentam 100\% de heterozigose, é maior que a produção dos grupos genéticos parentais. Dessa forma, é possível que as vacas com maiores heterozigoses produzam mais leite e proporcionem melhores condições para o desenvolvimento de seus bezerros.

Os critérios de ajuste obtidos para o modelo que considera o grupo genético do bezerro como efeito fixo classificatório (MSIM + CGG + INT) foram mais próximos de zero que aqueles obtidos para o modelo que utiliza a regressão múltipla para modelar os efeitos raciais e heteróticos (MSIM + RM + INT) (Tabela 2), sugerindo melhor ajuste do primeiro em relação ao último. Este resultado está de acordo com aqueles obtidos por Alencar et al. (1998) e Trematore et al. (1998), que avaliando a inclusão das composições genéticas e heterozigoses, individuais e maternas, como regressão múltipla (efeitos lineares) ou como sendo variáveis classificatórias, para análise do peso à desmama de bezerros cruzados CharolêsNelore, verificaram que apenas a inclusão dos coeficientes lineares para esses fatores não foi suficiente para explicar o comportamento da característica. Para explicar esta diferença no ajuste dos modelos, concorda-se com Trematore et al. (1998), que sugeriram que efeitos epistáticos, de ligação gênica e, ou não-lineares possam ser responsáveis por parte da variação existente entre grupos genéticos, para o peso à desmama de bezerros cruzados Charolês-Nelore. Efeitos significativos da epistasia e da complementaridade sobre características pré-desmama de bezerros cruzados Hereford-Nelore foram obtidos por Carvalheiro et al. (2006) e Pimentel et al. (2006).

No modelo que ajustou os efeitos genéticos aditivos e não-aditivos por meio de classes (MSIM + CGG + INT), apenas os dados pertencentes a uma classe são utilizados para estimar seu efeito. Isso pode gerar soluções incoerentes 
para as classes com número reduzido de dados, prejudicando a avaliação genética. No modelo que utilizou a regressão múltipla para aqueles efeitos genéticos (MSIM + RM + INT), os dados de todos os grupos genéticos podem contribuir para a obtenção das soluções e, assim, possibilitar a obtenção de soluções mais adequadas para alguns grupos genéticos, especialmente para aqueles com menor número de observações.

Independentemente da forma utilizada para o ajuste dos efeitos genéticos aditivos e não-aditivos médios, as estimativas de soluções para os bezerros do grupo A (5/16 Ch) foram superiores àquelas obtidas para os bezerros Canchim (filhos de vacas Canchim) (Tabela 3). Esses resultados aproximam-se daqueles reportados por Alencar et al. (1998) para bezerros 5/16 Charoleses, embora as magnitudes estimadas neste experimento sejam menores que aquela obtida pelos autores citados (12,8 kg). Parte da diferença em magnitude pode ser atribuída ao fato que os bezerros analisados por Alencar et al. (1998) eram filhos de vacas 5/8 Charolesas, com 75\% de heterozigose materna, enquanto os bezerros deste estudo eram filhos de vacas Canchim, com aproximadamente $62,5 \%$ de heterozigose.

Como os bezerros T1 (15/32 Ch) foram provenientes de cruzamentos absorventes de vacas Zebus com touros Canchim, seriam esperadas diferenças pequenas entre as soluções deste grupo e aquela do grupo Canchim filhos de vacas V (9/16 Ch), o que pode ser observado na análise das soluções obtidas com o modelo que considerou a regressão múltipla para ajuste dos efeitos genéticos aditivos e nãoaditivos médios.

As soluções para os grupos genéticos V (9/16 Ch, filhos de vacas T1 ou T2) foram maiores que as do grupo Canchim (filhos de vacas Canchim), independentemente do modelo. As maiores heterozigoses, materna e individual, podem ter contribuído para essas superioridades. As soluções para o grupo $\mathrm{V}$ (filhos de vacas $1 / 2 \mathrm{Ch}$ ) foram maiores que a do grupo $\mathrm{V}$ (filhos de vacas 15/32 Ch), mas as magnitudes dependeram do modelo considerado.

As superioridades das soluções para os bezerros $\mathrm{V}$ (filhos de vacas 1/2 Charolesas) foram de 12,887 e 2,226 kg em relação às soluções para os bezerros $\mathrm{V}$ (filhos de vacas 15/32 Charolesas) nos modelos com regressão múltipla ou classes de grupos genéticos, respectivamente. Alencar et al. (1998), comparando o desempenho de bezerros com a mesma composição genética individual, 1/4 Ch e 50\% de heterozigose, filhos de vacas com $0 \%$ ou $100 \%$ de heterozigose, Nelore ou F1 Charolês-Nelore, estimaram que os bezerros filhos das vacas $\mathrm{F} 1$ foram 10,8 kg mais pesados à desmama que os filhos das vacas Nelore. Esta superioridade pode ser atribuída ao efeito da heterozigose sobre a produção de leite das vacas mestiças (Cerdótes et al., 2004), e não às diferenças raciais. Dessa forma, o modelo que ajustou os efeitos aditivos e não-aditivos por meio de regressão múltipla foi capaz de proporcionar resultados mais próximos dos esperados para os animais do grupo V. Outro fato que provavelmente contribuiu para esta superioridade do modelo com regressão múltipla, em relação ao modelo com classes de grupos genéticos, é que, para a obtenção das soluções no modelo de classes foram utilizados, majoritariamente, dados de fêmeas do grupo $\mathrm{V}$, pois poucos dados de machos estavam disponíveis para análise.

Comparando-se os bezerros 5/8 Charoleses com os bezerros Canchim (filhos de vacas Canchim), percebe-se que as maiores diferenças estão relacionadas aos percentuais de heterozigose individual e de alelos da raça Charolesa na mãe (Tabela 1). Estas diferenças poderiam proporcionar diferenças no desempenho dos animais na

Tabela 3 - Estimativas das soluções para cada grupo genético (efeitos genéticos aditivos diretos e maternos + heteroses individual e materna) em função do tipo de modelagem para os efeitos da composição genética (percentual de alelos da raça Charolesa e de heterozigose) individual e materna, em relação às estimativas dos bezerros Canchim (filhos de vacas Canchim)

\begin{tabular}{|c|c|c|c|}
\hline \multicolumn{2}{|c|}{ Grupo genético } & \multicolumn{2}{|c|}{ Soluções (kg) } \\
\hline Bezerro & Vaca & Regressão múltipla & Classes de grupos genéticos \\
\hline $1 / 4 \mathrm{Ch}$ & T1 (15/32 Ch) & $-13,741$ & 14,446 \\
\hline A $(5 / 16 \mathrm{Ch})$ & Canchim & 2,761 & 0,959 \\
\hline T1 (15/32 Ch) & A $(5 / 16 \mathrm{Ch})$ & 2,347 & 7,729 \\
\hline V (9/16 Ch) & T1 (15/32 Ch) & 1,196 & 4,031 \\
\hline Canchim & V (9/16 Ch) & 0,427 & 0,504 \\
\hline Canchim & $5 / 8 \mathrm{Ch}$ & 7,881 & 10,792 \\
\hline Canchim & Canchim & 0,000 & 0,000 \\
\hline Canchim & MA (21/32 Ch) & 6,144 & 12,299 \\
\hline MA (21/32 Ch) & A $(5 / 16 \mathrm{Ch})$ & 22,907 & 21,478 \\
\hline
\end{tabular}

${ }^{1} \mathrm{Ch}=$ Charolês; $\mathrm{Z}=$ Zebu. 
ordem de 20,1 $\mathrm{kg}$ a $23,5 \mathrm{~kg}$ a favor dos bezerros 5/8 Charoleses. Diferenças entre estes grupos genéticos poderiam ser explicadas pela heterose residual nas gerações avançadas de cruzamentos. Resultados teóricos para raças compostas semelhantes à Canchim, apresentados por Barbosa (1995) indicam que a heterose residual na geração F3 é de 46,9\%. Não foram encontrados resultados experimentais sobre heterose residual em cruzamentos de duas raças. Todavia, os resultados experimentais para heterose residual sobre o peso aos 200 dias de idade de bovinos na geração $\mathrm{F} 3$ em cruzamentos de quatro raças não foram diferentes $(\mathrm{P}>0,05)$ dos valores teóricos (Gregory et al., 1991), confirmando a possibilidade da utilização deste modelo para explicação das diferenças entre os grupos 5/8 Ch e Canchim (filhos de vacas Canchim).

Em relação às soluções para os grupos Canchim filhos de vacas V ou 5/8 Charolesas, não houve diferenças marcantes entre as opções para modelagem dos efeitos aditivos e não-aditivos médios. As estimativas das diferenças de desempenho para os bezerros Canchim filhos de vacas V, em relação aos bezerros Canchim filhos de vacas Canchim, foram menores que entre os bezerros Canchim filhos de vacas 5/8 Charolesas em relação aos bezerros Canchim filhos de vacas Canchim. Nestes casos, os bezerros possuem composições genéticas e níveis de heterozigose semelhantes, enquanto suas mães são diferentes, especialmente quanto à heterozigose. Considerando a associação positiva entre heterozigose e produção de leite da vaca (Cerdótes et al., 2004), a maior heterozigose das vacas $5 / 8$ Charolesas (75\%) indica sua capacidade de produzir mais leite que as concorrentes V e Canchim, corroborando o maior peso de suas crias à desmama.

Para os bezerros Canchim, filhos de vacas MA (21/32 Ch), os resultados seguem a tendência esperada, de maior peso à desmama em relação aos bezerros Canchim filhos de vacas Canchim, possivelmente em função de fatores já mencionados, como maiores percentuais de alelos da raça Charolesa individual e materno e maior percentual de heterozigose materna. Contudo, a diferença estimada considerando-se as classes de grupos genéticos foi superior àquela estimada a partir do modelo de regressão múltipla. Ao utilizarem os coeficientes de regressão estimados por Trematore et al. (1998) para as mesmas covariáveis consideradas neste trabalho e para a característica peso à desmama de bezerros Charolês-Nelore, a superioridade de desempenho dos animais Canchim filhos de vacas MA, em relação aos Canchim filhos de vacas Canchim, seria de $8,683 \mathrm{~kg}$. Este valor está entre as soluções obtidas pelos modelos classificatório e de regressão múltipla, contudo mais próximo do último.
O desempenho dos animais do grupo genético MA (21/32 Charolês) foi superior ao dos animais Canchim em aproximadamente $22 \mathrm{~kg}$. Possivelmente, os maiores percentuais de alelos da raça Charolesa e de heterozigose individuais justificam estas diferenças. Este resultado está de acordo com aqueles apresentados por Ipameri Agropecuária (2003), uma vez que a superioridade dos animais MA para a característica peso à desmama (231 dias) foi de aproximadamente $14 \mathrm{~kg}$, em relação aos Canchim provenientes de diferentes esquemas de cruzamentos, considerando-se uma amostra de machos selecionados.

Considerando os resultados disponíveis na literatura e o disposto anteriormente, nota-se que as diferenças entre os grupos genéticos foram melhor estimadas com a utilização do modelo de regressão múltipla (MSIM + RM + INT) em relação ao modelo com classes de grupos genéticos (MSIM + CGG + INT).

A inclusão da interação grupo genético $\times$ grupo de contemporâneos proporcionou redução nas variâncias genéticas aditivas direta e materna (Tabela 4) e, consequentemente, as herdabilidades também diminuíram. Isto pode indicar que as variâncias genéticas aditivas e herdabilidades estavam sendo inflacionadas nos modelos que não contemplaram a interação. Uma das consequências da superestimação das variâncias genéticas aditivas e herdabilidades, quando a seleção é baseada nos BLUP, é a tendência em atribuir maior importância às informações dos próprios animais em relação ao pedigree e aos dados da progênie (Carneiro et al., 2001), reduzindo a eficiência da identificação dos potenciais genéticos.

As variações observadas nas estimativas dos parâmetros, em razão de considerar a regressão múltipla ou o efeito classificatório para modelar os efeitos genéticos aditivos e não-aditivos médios, foram de pequena magnitude. Isso sugere que aqueles fatores genéticos nãoaditivos (epistasia e ligação gênica), que provavelmente proporcionaram ajustes estatisticamente diferentes para os modelos, não foram suficientes para causar alterações de grande magnitude nas estimativas dos componentes de covariância.

Quando o grupo genético do animal foi concatenado ao grupo de contemporâneos, houve redução nas variâncias genéticas aditivas e fenotípica e aumento na variância de ambiente materno permanente e residual, em relação aos demais modelos. Isto sugere que parte da variância da interação grupo genético × grupo de contemporâneos estava sendo absorvida pelas variâncias genéticas e inflacionando as estimativas de herdabilidade, semelhante ao observado por Alencar et al. (2005) para os pesos à desmama e aos doze meses de bovinos Canchim. 
Tabela 4 - Estimativas de parâmetros ${ }^{1}$ para o peso à desmama de bezerros mestiços Charolês-Zebu, de acordo com o modelo estatístico

\begin{tabular}{|c|c|c|c|c|c|c|c|}
\hline & & & & Iodelo estatísti & & & \\
\hline & MSIM & MSIM + INT & MSIM + RM & MSIM + CGG & MSIM + RM + INT & MSIM + CGG + INT & MUTIL \\
\hline$\partial_{a}^{2}$ & 155,46 & 131,03 & 144,72 & 144,05 & 124,67 & 126,15 & 110,70 \\
\hline$\partial_{m}^{2}$ & 63,14 & 54,34 & 56,89 & 52,22 & 50,25 & 47,74 & 43,34 \\
\hline$\partial_{a m}$ & $-48,49$ & $-38,94$ & $-52,24$ & $-48,12$ & $-42,67$ & $-40,63$ & $-34,10$ \\
\hline $\mathrm{\partial}_{p}^{2}$ & 107,20 & 110,35 & 113,71 & 113,22 & 115,20 & 114,64 & 116,25 \\
\hline $\mathrm{\delta}_{q}^{2}$ & - & 41,25 & - & - & 36,53 & 33,38 & - \\
\hline$\partial_{e}^{2}$ & 479,66 & 478,24 & 484,83 & 484,53 & 482,01 & 481,32 & 488,68 \\
\hline$\partial_{f}^{2}$ & 756,96 & 776,27 & 747,92 & 745,91 & 765,99 & 762,61 & 724,87 \\
\hline$\hat{h}^{2}$ & 0,21 & 0,17 & 0,19 & 0,19 & 0,16 & 0,17 & 0,15 \\
\hline $\mathrm{m}^{2}$ & 0,08 & 0,07 & 0,08 & 0,07 & 0,07 & 0,06 & 0,06 \\
\hline$\hat{r}_{a m}$ & $-0,49$ & $-0,46$ & $-0,58$ & $-0,55$ & $-0,54$ & $-0,52$ & $-0,49$ \\
\hline$\hat{p}^{2}$ & 0,14 & 0,14 & 0,15 & 0,15 & 0,15 & 0,15 & 0,16 \\
\hline$q^{2}$ & - & 0,05 & - & - & 0,05 & 0,04 & - \\
\hline$\hat{e}^{2}$ & 0,63 & 0,62 & 0,65 & 0,65 & 0,63 & 0,63 & 0,67 \\
\hline
\end{tabular}

De modo geral, os valores de herdabilidade direta obtidos no presente estudo, que variaram entre 0,15 e 0,21 , foram inferiores aos valores de 0,48 e 0,35 estimados por Mello et al. (2002) e Toral et al. (2007), respectivamente, mas semelhantes ao valor de 0,17 , obtido por Barichello et al. (2010), que utilizaram apenas os dados de animais da raça Canchim, filhos de vacas Canchim. As estimativas da herdabilidade materna, que neste estudo variaram entre 0,06 e 0,08 , foram superiores ao valor de 0,04 estimado por Mello et al. (2002) e semelhante à estimativa de 0,09 obtida por Barichello et al. (2010). É possível que além das diferenças entre as bases de dados, a inclusão dos efeitos de grupos genéticos tenha contribuído para a obtenção de valores diferentes. Estas estimativas de herdabilidade indicam que o cruzamento e a seleção podem ser utilizados como ferramentas para modificar a composição genética dos rebanhos, no sentido de aumentar o peso à desmama.

As proporções da variância fenotípica, atribuídas ao efeito de ambiente materno permanente, variaram entre 0,14 e 0,16 , valores semelhantes ao de 0,14 , estimado por Mello et al. (2002), evidenciando a importância destes efeitos para a expressão do peso à desmama dos bezerros. A correlação entre os efeitos genéticos aditivos direto e materno variou entre-0,58 e-0,46. Mercadante \& Lôbo (1997), trabalhando com dados de bezerras Nelore, bem como Mello et al. (2002), trabalhando com dados de bezerros machos e fêmeas da raça Canchim, obtiveram valores próximos a zero para esta correlação. Essas diferenças podem ser atribuídas, em parte, às diferenças na estrutura dos dados, no número de mães e de avós com seus próprios dados de desempenho, sendo que, quanto maior for o número de mães e avós com seus próprios dados e o número de filhos por mãe, melhores serão as estimativas (Maniatis \& Pollott, 2003).

Conforme demonstrado nos testes estatísticos, a inclusão da interação entre grupo genético e grupo de contemporâneos proporcionou melhoria no ajuste dos modelos e foi responsável por aproximadamente 5\% da variância fenotípica. Este efeito aleatório pode ser visto como uma alternativa a ser considerada nas avaliações genéticas de animais mestiços para modelar o efeito da interação genótipo $\times$ ambiente e diferenças de manejo não registradas e dadas aos animais de grupos genéticos diferentes e criados na mesma fazenda.

De modo geral, os diferentes modelos proporcionaram partições diferentes apenas para as covariâncias genéticas e fenotípicas, não provocando alterações nas variâncias residuais, que representaram entre $62 \%$ e $67 \%$ da variância fenotípica. Estes valores sugerem uma possível ocorrência de outros fatores ambientais e, ou genéticos, que podem influenciar o peso à desmama nessa população e que não foram considerados nos modelos utilizados, como interações touro $\times$ fazenda e touro $\times$ grupo de contemporâneos.

Em análises posteriores, as correlações de Spearman entre os valores genéticos aditivos preditos dos touros com pelo menos um filho nascido e avaliado entre os anos 2000 e 2005, a partir dos três modelos com melhor ajuste (Tabela 2), foram calculadas.

Considerando os valores genéticos preditos dos touros obtidos das análises com regressão múltipla ou classes de grupos genéticos para modelagem dos efeitos genéticos aditivos e não-aditivos médios, as correlações de Spearman foram de 0,99 e 1,00 para touros mestiços e Charoleses, 
respectivamente. Estes resultados sugerem que a utilização de diferentes alternativas para modelagem dos efeitos genéticos aditivos e não-aditivos médios têm pouca influência sobre as classificações dos touros.

Correlações de Spearman de 0,93 e 0,95 (touros mestiços) e de 0,78 e 0,77 (touros Charoleses) foram obtidas para os valores genéticos preditos pelo modelo semelhante ao utilizado na avaliação genética da raça Canchim (MUTIL) com os valores preditos nos modelos com regressão múltipla ou classes de grupos genéticos para modelagem dos efeitos genéticos aditivos e não-aditivos médios, respectivamente. Este resultado pode ser considerado relevante porque implicaria em alterações na classificação dos reprodutores.

Com base nos critérios de ajuste utilizados neste trabalho, especialmente o CAIC (Tabela 2), e na interpretação biológica dos resultados, os modelos considerando a interação entre grupo genético e grupo de contemporâneos e a modelagem dos efeitos genéticos aditivos e não-aditivos médios por meio de regressão múltipla ou classes de grupos genéticos demonstraram superioridade, em relação ao modelo MUTIL. Além disso, com a utilização do modelo de regressão múltipla, seria possível obter soluções mais adequadas, do ponto de vista biológico, para grupos genéticos com menor número de observações, bem como ajustar as DEPs de tal modo que reprodutores de diferentes grupos genéticos, mas que são utilizados para a produção de animais Canchim, possam ser comparados sob a pressuposição de homogeneidade de variâncias. Contudo, a possibilidade de existência de variâncias genéticas diferentes entre grupos genéticos analisados em conjunto também requer estudos antes da implementação de qualquer modelo para avaliação genética de populações cruzadas.

\section{Conclusões}

A interação entre o grupo genético do bezerro e o grupo de contemporâneos é uma fonte de variação importante e a sua inclusão nos modelos de avaliação genética de bezerros Charolês-Zebu, para o peso à desmama, pode contribuir para a identificação do potencial genético dos reprodutores, a partir da utilização de dados de campo. Os efeitos genéticos aditivos e não-aditivos médios do bezerro e da vaca podem ser modelados por meio da utilização de regressão múltipla.

\section{Referências}

ALENCAR, M.M.; TREMATORE, R.L.; OLIVEIRA, J.A.L. Características de crescimento até a desmama de bovinos da raça Nelore e cruzados Charolês-Nelore. Revista Brasileira de Zootecnia, v.27, n.1, p.40-46, 1998.
ALENCAR, M.M.; MASCIOLI, A.S.; FREITAS, A.R. Evidências de interação genótipo $\mathrm{x}$ ambiente sobre características de crescimento em bovinos de corte. Revista Brasileira de Zootecnia, v.34, n.2, p.489-495, 2005.

ASSOCIAÇÃO BRASILEIRA DE CRIADORES DE CANCHIM ABCCAN. Embrapa-Geneplus. Sumário de touros Canchim, MA e Charolês. Campo Grande: Geneplus Consultoria Agropecuária Ltda, 2004.

ASSOCIAÇÃO BRASILEIRA DE CRIADORES DE CANCHIM ABCCAN. [2008]. Novos esquemas de acasalamento para obtenção do Canchim. Disponível em: <http://www.canchim.com.br/ raca.asp\#esquemas> Acesso em: 18 jun. 2008.

BARBOSA, P.F. Heterose, heterose residual e efeitos da recombinação em sistemas de cruzamento de bovinos. Ribeirão Preto: Sociedade Brasileira de Genética, 1995. p.135-243. (Série monografias, número 2).

BARICHELLO, F.; ALENCAR, M.M.; TORRES JÚNIOR, R.A.A. et al. Herdabilidade e correlações quanto a peso, perímetro escrotal e escores visuais à desmama, em bovinos Canchim. Pesquisa Agropecuária Brasileira, v.45, n.6, p.563-570, 2010.

BOLDMAN, K.G.; KRIESE, L.A.; VAN VLECK, L.D. A manual for use of MTDFREML. A set of programs to obtain estimates of variance and covariance (DRAFT). Lincon: Department of Agriculture, Agricultural Research Service, 1995. 120p.

BOZDOGAN, H. Model selection and Akaike's information criterion (AIC): the general theory and its analytical extensions. Psychometrika, v.52, n.3, p.345-370, 1987.

CARNEIRO, A.P.S.; TORRES, R.A.; EUCLYDES, R.F. et al. Efeito da conexidade de dados sobre o valor fenotípico médio e a variância genética aditiva. Revista Brasileira de Zootecnia, v.30, n.2, p.336-341, 2001.

CARVALHEIRO, R.; PIMENTEL, E.C.G.; CARDOSO, V. et al. Genetic effects on preweaning weight gain of Nelore-Hereford calves according to different models and estimation methods. Journal of Animal Science, v.84, n.11, p.2925-2933, 2006.

CERDÓTES, L.; RESTLE, J.; ALVES FILHO, D.C. et al. Produção e composição do leite de vacas de quatro grupos genéticos submetidas a dois manejos alimentares no período de lactação. Revista Brasileira de Zootecnia, v.33, n.3, p.610-622, 2004

GREGORY, K.E.; CUNDIFF, L.V.; KOCK, R.M. Breed effects and heterosis in advanced generations of composite populations for growth traits in both sexes of beef cattle. Journal of Animal Science, v.69, n.8, p.3202-3212, 1991.

HENDERSON, C.R.; KEMPTHORNE, O.; SEARLE, S.R. et al. The estimation of environmental and genetic trends from records subject to culling. Biometrics, v.15, n.2, p.192-218, 1959.

IPAMERI AGROPECUÁRIA. Programa de avaliação de touros a campo Canchim Ipameri. 6.ed. Jussara: Ipameri Agropecuária, 2003. 22p.

MANIATIS, N.; POLLOTT, G.E. The impact of data structure on genetic (co)variance components of early growth in sheep, estimated using an animal model with maternal effects. Journal of Animal Science, v.81, n.1, p.101-108, 2003.

MELLO, S.P.; ALENCAR, M.M.; SILVA, L.O.C. et al. Estimativas de (co)variâncias e tendências genéticas para pesos em um rebanho Canchim. Revista Brasileira de Zootecnia, v.31, n.4, p.1707-1714, 2002.

MERCADANTE, M.E.Z.; LÔBO, R.B. Estimativas de (co)variâncias e parâmetros genéticos dos efeitos direto e materno de características de crescimento de fêmeas de um rebanho Nelore. Revista Brasileira de Zootecnia, v.26, n.6, p.1124-1133, 1997.

NEWMAN, S.; MAcNEIL, M.D.; REYNOLDS, W.L. et al. Fixed effects in the formation of a composite line of beef cattle: II pre- and postweaning growth and carcass composition. Journal of Animal Science, v.71, n.8, p.2033-2039, 1993.

PAZ, C.C.P.; ALBUQUERQUE, L.G.; FRIES, L.A. Efeitos ambientais sobre ganho de peso no período do nascimento ao desmama em bovinos da raça Nelore. Revista Brasileira de Zootecnia, v.28, n.1, p.55-64, 1999. 
PIMENTEL, E.C.G.; QUEIROZ, S.A.; CARVALHEIRO, R. et al. Estimativas de efeitos genéticos em bezerros cruzados por diferentes modelos e métodos de estimação. Revista Brasileira de Zootecnia, v.35, n.3, p.1020-1027, 2006 (supl.).

ROSO, V.M.; FRIES, L.A. Avaliação das heteroses materna e individual sobre o ganho de peso do nascimento ao desmame em bovinos Angus x Nelore. Revista Brasileira de Zootecnia, v.29, n.3, p.732-737, 2000.

SILVEIRA, J.C.; McMANUS, C.; MASCIOLI, A.S. et al. Fatores ambientais e parâmetros genéticos para características produtivas e reprodutivas em um rebanho Nelore no Estado de Mato Grosso do Sul. Revista Brasileira de Zootecnia, v.33, n.6, p.1432-1444, 2004.

TORAL, F.L.B.; SILVA, L.O.C.; MARTINS, E.N. et al. Interação genótipo $\mathrm{x}$ ambiente em características de crescimento de bovinos da raça Nelore no Mato Grosso do Sul. Revista Brasileira de Zootecnia, v.33, n.6, p.1445-1455, 2004.

TORAL, F.L.B.; ALENCAR, M.M.; FREITAS, A.R. Abordagens frequentista e bayesiana para avaliação genética de bovinos da raça Canchim para características de crescimento. Revista Brasileira de Zootecnia, v.36, n.1, p.43-53, 2007.

TORAL, F.L.B.; TORRES JÚNIOR, R.A.A.; LOPES, P.S. et al. Modeling the effect of the age of dam at calving on the weaning weight of Charolais-Zebu crossbred calves. Revista Brasileira de Zootecnia, v.38, n.7, p.1229-1237, 2009.

TORAL, F.L.B.; TORRES JÚNIOR, R.A.A.; LOPES, P.S. et al. Modeling the effect of age of dam at calving $x$ breed group of dam's interaction on weaning weight of Charolais-Zebu crossbred calves. Revista Brasileira de Zootecnia, v.39, n.5, p.1046-1057, 2010.

TREMATORE, R.L.; ALENCAR, M.M. BARBOSA, P.F. et al. Estimativas de efeitos aditivos e heteróticos para características de crescimento pré-desmama em bovinos Charolês-Nelore. Revista Brasileira de Zootecnia, v.27, n.1, p.87-94, 1998.

WOLFINGER, R. Covariance structure selection in general mixed models. Communications in Statistics - Simulation, v.22, n.4, p.1079-1106, 1993. 